\title{
Influence of Stuttering Therapy on Clinicians' Disfluencies: Effects of Client Modeling
}

\author{
Michael J. Kimbarow \\ University of Minnesota, Minneapolis, Minnesota \\ and \\ David A. Daly \\ University of Michigan, Ann Arbor, Michigan
}

\begin{abstract}
This study investigated whether student clinicians working with stutterers subsequently produce more disfluencies than student clinicians providing therapy to clients with other speech and language disorders. Seventeen graduate students working in a 6-wk summer camp setting were divided into two groups: eight who provided treatment for stutterers (group 1) and nine who provided therapy for clients with other communication disorders (group 2). All student clinicians were recorded during spontaneous speaking and oral reading tasks prior to camper arrival and following camper departure. An eight-category classification system was used to determine disfluency types. Findings revealed that Group 1 clinicians significantly decreased their total disfluencies between pre- and post-camp recordings on the spontaneous speaking task. Unexpectedly, this same group also substantially increased part-word repetitions and sound prolongations. The possibilities of incidental learning, reverse modeling, and overidentification with stuttering clients are discussed.
\end{abstract}

\section{INTRODUCTION}

The effect of modeling procedures with stutterers has been actively investigated by Gregory and his students for more than a decade (1968). In his carefully designed step-by-step stuttering therapy program, Gregory $(1973 a, b)$ emphasized the clinician's role as a modeler, demonstrating the skills and rewarding the client whenever desired behaviors are approximated. Gregory maintains that instructing disfluent children to

Address correspondence to: David A. Daly, Ed.D., Speech and Hearing Sciences, University of Michigan, 1111 E. Catherine Street, Ann Arbor, MI 48109. 
imitate the easy relaxed speech of their clinicians is highly successful in treating stuttering.

The effect of modeling on the frequency of stuttering has also been explored by Martin and Haroldson (1977) as a part of a larger study on vicarious punishment. After viewing a videotape of a severe stutterer being punished for his disfluencies, 20 stuttering subjects exhibited a significant reduction in their own subsequent disfluency rates. In summarizing their findings, Martin and Haroldson (1977) noted, "The results suggest that a client may realize some reduction in stuttering frequency simply by observing another client responding dramatically to a treatment procedure" (p. 25).

If the frequency of stuttering is effected by clinician or peer modeling, it would seem plausible that interaction between clinicians and their clients who stutter might also have the potential to effect a change in the fluency of the clinicians. Students, who provide therapy for stutterers, frequently express sincere concerns to their supervisors when they suspect that their speech is becoming increasingly more disfluent. Clinical supervisors, not always knowing whether the concerns are justified or not, typically reassure student clinicians that such changes are normal and, in fact, expected. A review of the literature revealed a paucity of information in this area. No data could be located to determine whether clinicians' reports of increased disfluencies were real or imagined.

The purposes of this study were to explore whether clinicians treating stuttering clients exhibit a change in their own disfluencies and whether the nature of these suspected changes could be identified.

\section{METHOD}

Seventeen normal speaking female graduate students in speech and language pathology attending the 1976 session of the University of Michigan's Shady Trails Camp served as subjects. The 17 students ranged in age from 21-24 yr, with a mean age of $23.4 \mathrm{yr}$. Following clinical assignments, the students were divided into two groups. Group 1 consisted of eight clinicians who were actively involved in the implementation of an intensive fluency shaping program at camp for the stutterers. Group 2 consisted of the nine remaining student clinicians who provided speech and language for clients exhibiting disorders of articulation, 
language, voice, aphasia, cleft palate, etc. These nine graduate students had no direct therapeutic involvement with the disfluent campers.

During a precamper arrival orientation week, each of the 17 graduate students was individually audio-recorded on both spontaneous speaking and oral reading tasks. The eight-category classification system of Johnson et al. (1963) was used to determine disfluencies. The examiners' reliability of $91 \%$ in judging disfluencies of nonstuttering subjects has been reported elsewhere (Daly and Kimbarow, 1978).

Student clinicans were retested using the same procedures immediately following the $6 \mathrm{wk}$ of intensive therapy with either stuttering or nonstuttering speech and language handicapped children.

Riley's (1972) protocol for determining frequency of disfluencies in 100-word samples was followed. Students were asked to talk about a job for $3 \mathrm{~min}$ and then read aloud for the same time period. As Riley recommends, the first and last 25 words in each 150-word sample were not included in the frequency counts. The student clinicians' frequency of disfluencies were tabulated from the middle 100 words of their samples.

\section{RESULTS}

Table 1 shows the total, mean, and specific type of disfluencies during 100-word spontaneous speech samples for all clinicians. Interjections and whole-word repetitions were the most frequently observed disfluency types. Phrase repetitions and incomplete phrases did not occur in the speech samples analyzed. Precamp mean disfluency counts of 5.88 and 4.89 did not differ significantly between the two groups $(t=0.72, \mathrm{df}=$ $15, p=0.48$ ). Interestingly, a statistically significant reduction ( $p=0.05)$ in pre- versus postcamp disfluencies was obtained on a within group comparison for the eight group 1 clinicians involved in the fluency shaping program $(t=2.30, d f=7)$, but not on the within group comparison for the nine group 2 clinicians who treated clients with other types of speech and language disorders $(t=-1.03, d f=8, p=0.33$ ).

Inspection of Table 1 also reveals that group 1 clinicians reduced the frequency of their disfluencies in five of the eight categories. Marked increases occurred, however, on the two kernel characteristics of stuttering (after Wingate, 1964): part-word repetitions and sound prolongations. These specific increases are particularly noteworthy.

Two specific speech targets taught to stuttering clients by group 1 


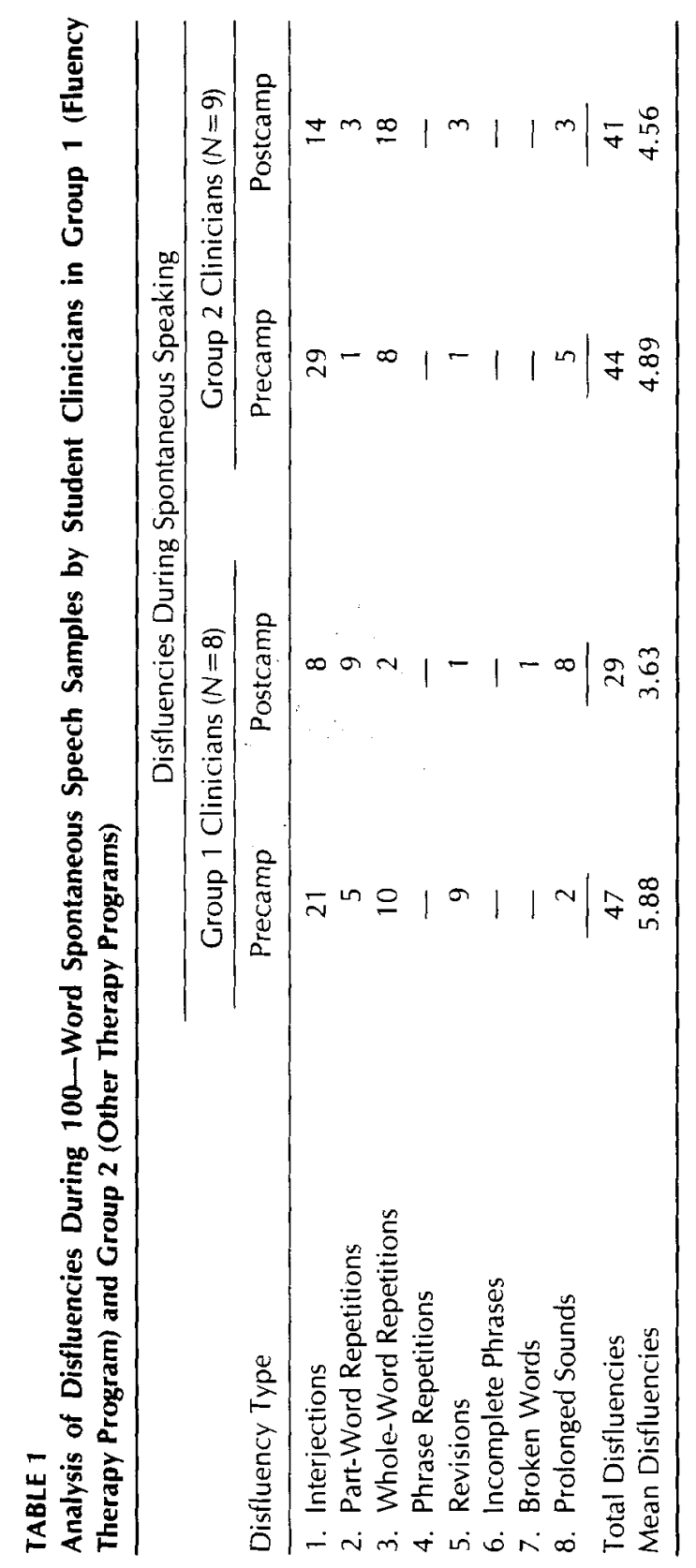


clinicians were stretched syllable speech and gentle onset (after Webster, 1975). These fluency shaping targets stress the gradual, easy prolongation of sounds. Continuous monitoring of these fluency shaping gestures in the stutterers' speech may have accounted for the increase in prolonged sounds shown for group 1 clinicians. Part-word repetitions quite possibly may have increased as a function of unconscious modeling or incidental learning.

Table 2 presents similar comparisons of disfluencies for both groups of clinicians during the oral reading task. As expected, far fewer disfluencies occurred during oral reading than during spontaneous speaking. As in the spontaneous samples, phrase repetitions and incomplete phrases did not occur during oral reading samples for any of the 17 subjects. Mean precamp disfluency counts of 0.75 and 1.78 were not statistically different $(p=0.12)$. However, the average increase of one disfluency between the pre- and postcamp comparisons on oral reading for group 1 clinicians was statistically significant $(t=-2.65, d f=7$, $p<0.05)$. A similar within-group comparison between pre- and postcamp disfluencies during oral reading did not reveal a significant difference for group 2 clinicians ( $p=0.33$ ).

Figure 1 illustrates the individual subject changes in disfluencies for student clinicians in both groups. Six of the group 1 clinicians (75\%) and six of the group 2 clinicians (66\%) decreased their disfluencies on the spontaneous speaking task. Unexpectedly, the greatest decrease and increase were made by group 2 clinicians, subject 9 and subject 15, respectively. No explanation is readily available to account for the dramatic shifts in disfluency between their pre- and postcamp samplings.

Figure 1 also shows that 11 of the 17 clinicians (65\%) increased their disfluencies on the oral reading task; six of eight students in group 1 and five of nine students in group 2. The high frequency of increased disfluencies during the postcamp oral reading task was unexpected and deserves further exploration.

\section{DISCUSSION}

Modeling has been shown to be an effective therapeutic technique to facilitate behavior change in a variety of settings (Heller and Marlatt, 1969; Gregory, 1968, 1973a,b; Bandura, 1974; Rimm and Masters, 


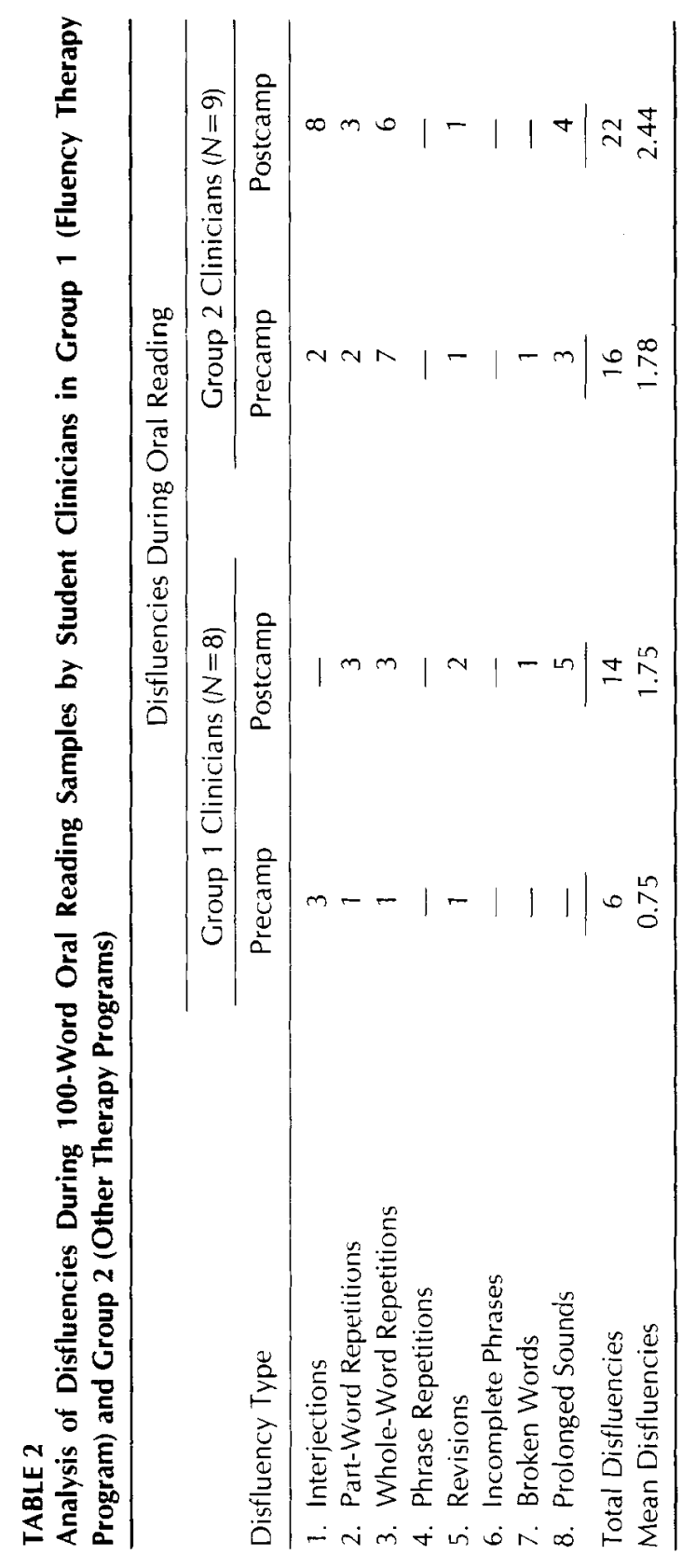




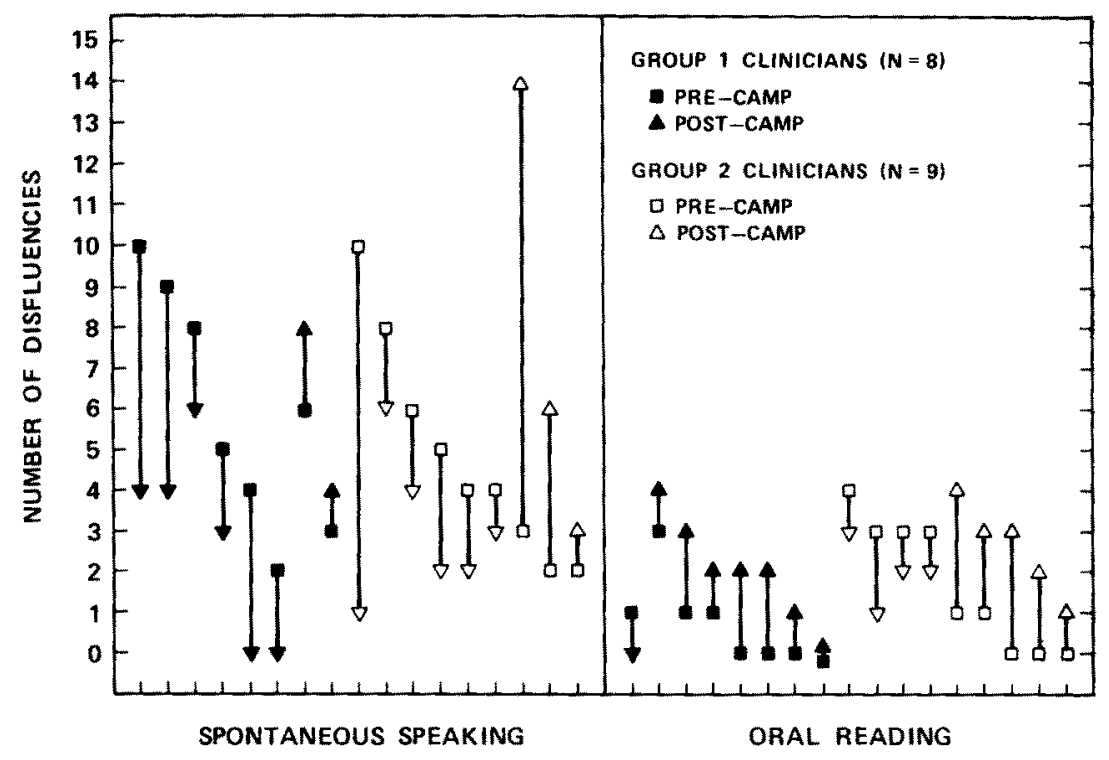

Figure 1. Individual subject pre- and postcamp disfluency counts and direction of change on spontaneous and oral reading tasks: group 1 (clinicians in fluency shaping program); group 2 (clinicians in other therapy programs).

1974; Martin and Haroldson, 1977). The present study was designed to take advantage of the unique potential which the camp environment offered for the study of such modeling procedures. In addition to meals and recreational events, the student clinicians spent a total of $4 \mathrm{hr} /$ day, 6 days/week for $6 \mathrm{wk}$ in direct clinical contact with their clients. This intensive environment would appear more than adequate for any potential client or clinician modeling to take place.

The group 1 clinicians were thoroughly instructed to serve as role models for their stuttering clients. Specifically, group 1 clinicians were taught to deliberately modify their own rate of speaking as well as teach vigilance of fluency control to their clients. This vigilance involved clinician and client monitoring of specific behaviors believed necessary to establish and maintain fluent speech. It is of interest to note that the stuttering youngsters enrolled in this intensive program did in fact achieve high levels of fluency at the end of therapy. It would appear that speech 
therapy techniques involving the imitation of their clinicians did result in the desired behavior change. The present data, however, raise the intriguing question of who was modeling whom? That is, could an imitative process, such as "reverse modeling" also effect the speech of the stutterers' clinicians?

Data clearly show that the group 1 clinicians increased the frequency of their prolongations and part-word repetitions between the preand posttherapy samplings. This shift from normal to abnormal disfluency types appears to reflect the clinicians' modeling of their clients' behavior. Although both clinicians and clients showed a decrease in the total number of disfluencies during spontaneous speaking at the end of camp, the continued presence of the kernel features of stuttering in the clients and the acquisition of these behaviors by the clinicians suggests that these features are resistant to modification through modeling techniques. This has interesting implications for therapy programs designed around modeling procedures and might possibly account for the typically poor maintenance of fluency reported in the literature.

The substantial increase in part-word repetitions and sound prolongations in the spontaneous speech of group 1 clinicians is not easily explained. Perhaps the constant exposure to these disfluency types in the speech of their stuttering clients simply elicited similar behaviors inherent in the verbal repertoires of the clinicians. Possibly the total acceptance of the disfluent campers and the absence of noxious feedback in the camp environment facilitated a release of these behaviors by the clinicians. Quite possibly, the residential nature of the intensive $6 \mathrm{wk}$ long therapeutic experience may account for the reverse modeling which apparently occurred. It is not uncommon for clinicians to consciously or unconsciously assume surrogate parent roles in this type of environment. Clinicians empathize and identify with the clients for whom they are responsible. Incidential learning, that is, learning that apparently takes place in the absence of any intent to learn specific behaviors, is a common phenomenon which has received much attention in the psychological literature (McGeoch and Irion, 1952; Bandura and Huston, 1961; Bandura, 1974). Quite possibly such incidential learning could explain the clearly identifiable increase in part-word repetitions and sound prolongations in the speech of the clinicians. Our knowledge of the 
factors, which facilitate observational or incidential learning in therapeutic settings, is scant. Imitation or reverse modeling should not be lightly dismissed as an unlikely explanation for student clinicians' increased disfluencies.

The postcamp increments in disfluencies during oral reading for both groups seem less troublesome to understand than the increases during spontaneous speaking. Possibly the familiarity of the reading material influenced the findings. All student clinicians read the Rainbow Passage (Fairbanks, 1960) during the precamp recording and a passage on the perception of clouds (Webster, 1975) during the postcamp assessment. While the passages were of comparable reading level, the Rainbow Passage was definitely more familiar to graduate students in specch and language pathology. Thus, familiarity of the reading material may have influenced the results.

Level of physical and mental alertness may account for the postcamp disfluency increments. All camp staff worked very long, arduous hours for a $7 \mathrm{wk}$ period. Fatigue certainly is an explanation that anyone who has worked at summer camps would not readily discount.

In a remarkably insightful chapter on the role of the clinician, Van Riper (1975) highlights several dimensions which might be explored in studying the stutterer's clinician. In describing numerous characteristics he specifically discusses the need for empathy, but cautions clinicians about overidentifying with their stuttering clients. Identification with clients is an aspect of treatment which deserves further study. The possible transference of disfluencies appears intrinsically related to the process of identification. Further research would be useful to determine whether similar increases in disfluencies occur in other clinicians practicing stuttering therapy in other settings. We simply do not know whether other clinicians who treat stuttering clients also acquire abnormal disfluencies. Further research would be useful in order to determine whether the amount of client contact is related to such changes, or whether such changes are short term or permanent. Longitudinal studies which record clinicians at specified intervals during therapy, as well as before and after, and which would examine factors such as frequency and duration of treatment and type of fluency program used, may shed considerable light on this intriguing phenomenon. 
The authors are grateful to the staff at Shady Trails Camp for their cooperation during the investigation. This paper is a revision of a report presented at the 1978 Convention of The American Speech-LanguageHearing Association.

\section{REFERENCES}

Bandura, A. (Ed.) Psychological Modeling: Conflicting Theories. New York: Atherton Company, 1974.

Bandura, A. and Huston, A.C. Identification as a process of incidental learning. Journal of Abnormal and Social Psychology, 1961, 63, 311-318.

Daly, D.A., and Kimbarow, M.L. Stuttering as operant behavior: Effect of the verbal stimuli "wrong", "right", and "tree" on the disfluency rates of school-age stutterers and nonstutterers. Journal of Speech and Hearing Research, 1978, 21, 589-597.

Fairbanks, G. Voice and Articulation Drillbook. New York: Harper and Brothers, 1960.

Gregory 11. Applications of learning theory concepts in the management of stuttering. In H. Gregory (Ed.) Learning Theory and Stuttering Therapy. Evanston, IL: Northwestern University Press, 1968.

Gregory, H. Modeling procedures in the treatment of elementary school children who stutter. Journal of Fluency Disorders, 1973a, 1, 58-63.

Gregory, H. Stuttering: Differential Evaluation and Therapy. New York: BobbsMerrill, 1973b.

Heller, K., and Marlatt, G.A. Verbal conditioning, behavior therapy, and behavior change: Some problems in extrapolation. In Cyril M. Franks (Ed.), Behavior Therapy: Appraisal and Status. New York: McGraw-Hill, 1969.

Johnson, W., Darley, F., and Spriesterbach, D. Diagnostic Methods in Speech Pathology. New York: Harper \& Row, 1963.

Martin, R. and Haroldson, S. Effect of vicarious punishment on stuttering frequency, Journal of Speech and Hearing Research, 1977, 20, 21-26.

McGeoch, J.A., and Irion, A.L. The Psychology of Human Leaning. New York: Longmans, Green, 1952.

Kiley, G. A stuttering severity instrument for children and adults. Journal of Speech and Hearing Disorders, 1972, 37, 314-322.

Rimm, D.C., and Masters, J.C. Behavior Therapy. New York: Academic Press, 1974.

Van Riper, C. The stutterer's clinician. In J. Eisenson (Ed.) Stuttering: A Second Symposium. New York: Harper \& Row, 1975.

Webster, R.L. Clinician's Program Guide: The Precision Fluency Shaping Guide. Roanoke, VA.: Communications Development Corporation, 1975.

Wingate, M.E. A standard definition of stuttering. Journal of Speech \& Hearing Disorders, 1964, 29, 484-489. 